Divide throughout by $2 R \cos A \cos B \cos C$, $\therefore \tan A+\tan B+\tan C=\tan A \tan B \tan C$,

A. G. Burgess.

To draw a circle which cuts three circles at the ends of the diameters of these circles.- Let the three circles have centres, $A, B, C$, and let their radical centre be $T$, and the

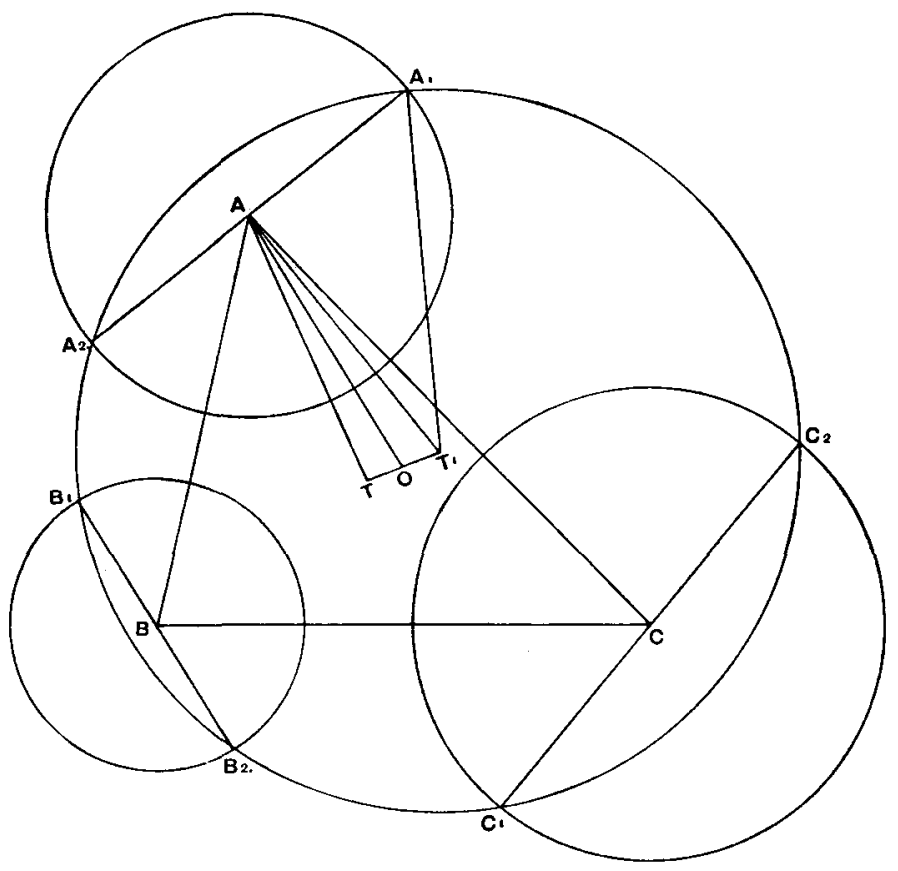

circumcentre of $\triangle A B C$ be $O$. Join $T O$ and produce it to $T_{1}$, so that $T_{1} O=T O . \quad T_{1}$ is the centre of the required circle. Join $T_{1} A$ and $T_{1} C$, and draw diameters $A_{1} A A_{2}$, and $C_{1} C C_{2}$ perpendicular to $T_{1} A$ and $T_{1} C . \odot$ with centre $T_{1}$ and radius $T_{1} A_{2}$ passes through $A_{1}$. 
DISCRIMINation OF the ROOTN OF a CUBIC EQUATION, RTC.

$$
\begin{aligned}
A_{2} T_{1}^{2}=A T_{1}^{2}+A A_{2}^{2} & =A T_{1}^{2}+A T^{2}-\left(A T^{2}-A A_{2}^{2}\right) \\
& =2 O A^{2}+2 O T^{2}-\left(C T^{2}-C C_{1}^{2}\right)
\end{aligned}
$$

( $T$ is radical centre

$$
\begin{aligned}
& \left.\quad \therefore A T^{2}-A A_{2}{ }^{2}=C T^{2}-C C_{1}{ }^{2}\right) \\
= & 2 O C^{2}+2 O T^{2}-\left(C T^{2}-C C_{1}{ }^{2}\right) \\
= & C T_{1}{ }^{2}+C T^{3}-C T^{2}+C C_{1}{ }^{2} \\
= & C T_{1}^{2}+C C_{1}{ }^{2} \\
= & C_{1} T_{1}{ }^{2} .
\end{aligned}
$$

$\therefore \quad \odot$ with centre $T_{1}$ and radius $A_{2} T_{1}$ passes through $C_{1}$ and hence through $C_{2}$.

Similarly, it will pass through the ends of the diameter perpendicular to $T_{1} B$.

If $\rho$ be the radius of radical circle, and $R$ of circumcircle of $\triangle A B C$,

$$
\begin{array}{ll} 
& A_{2} T_{1}^{2}=A T_{1}^{2}+A A_{2}^{2}, \text { and } \rho^{2}=A T^{2}-A A_{2}{ }^{2} \\
\therefore \quad & A_{2} T_{1}^{2}+\rho^{2}=A T^{2}+A T_{1}{ }^{2}=2 O A^{2}+2 O T^{2} \\
& \therefore \quad A_{2} T_{1}{ }^{2}=2 R^{2}+2 O T^{2}-\rho^{2} .
\end{array}
$$

A. G. Burgess.

\section{Discrimination of the Roots of a Cubic Equation by} Elementary Algebra. - The following note shows how the conditions for the reality or equality of the roots of a cubic can be obtained from the similar conditions for a quadratic. The method does not involve the use of the calculus or the properties of turning points, nor of the imaginary cube roots of unity.

Suppose the general cubic equation has been reduced as usual to the form

$$
x^{3}+p x+q=0 .
$$

It is certain that this has at least one real root, $\alpha$, say : reduce all the roots of the equation by $\alpha$ and we get

$$
\begin{array}{rlrl}
(\xi+\alpha)^{3}+p(\xi+\alpha)+q & =0, \\
\text { or, } \quad & \left(\alpha^{3}+p \alpha+q\right)+\left(\xi^{3}+3 \alpha \xi^{2}+\overline{3 \alpha^{2}+p} \xi\right) & =0, \\
\text { or, } \quad & \xi\left(\xi^{2}+3 \alpha \xi+3 \alpha^{2}+p\right) & =0,
\end{array}
$$

so that the roots of (1) are $\alpha, \alpha+\xi_{1}, \alpha+\xi_{2}$, where $\xi_{1}, \xi_{2}$ are the roots of

$$
\xi^{2}+3 \alpha \xi+\overline{3 \alpha^{2}+p}=0
$$

